\title{
Photonic Materials by the Sol-Gel Process
}

\author{
Jeffrey I. ZINK and Bruce S. DUNN* \\ Department of Chemistry and Biochemistry, University of California, Los Angeles, Los Angeles, CA 90024, USA \\ *Department of Materials Science and Engineering, University of California, Los Angeles, Los Angeles, CA 90024, USA
}

\section{ゾル-ゲル法によるフォトニックス材料}

\begin{abstract}
Jeffrey I. Zink and Bruce S. Dunn*
Department of Chemistry and Biochemistry, University of California, Los Angeles, Los Angeles, CA 90024, USA

*Department of Materials Science and Engineering, University of California, Los Angeles, Los Angeles, CA 90024, USA

ゾルーゲル法により任意に選んだ光学的性質を持つ有機分子を含有した非晶質無機固体の低温合成が可能である. 有機分子は熱的にはあまり安定性がなく，そのために高温で合成される一般の酸化物を母体として含有させるこ とはできなかった．ゾル-ゲル法を利用することにより，多種類の有機分子をゾル-ゲル母体中に含有させること ができ，その有機分子の特定の光学的性質をゾル-ゲルの状態に扔いても保持していることが示された．したが って，ゾルーゲル法により蛍光やフォトクロミック特性，非線型光学特性，レーザー作用などを持つフォトニッ クス材料を合成することができる．この報文においてはゾルーゲル法により有機物を含有したフォトニックス材 料を合成した 2 種類の分野の解説をする. その第 1 の分野はゾルーゲル反応過程を調べるために有機物添加体が 蛍光体として利用されるものである. 反応固体の剛性や溶媒の化学的変化の例を示す. 第 2 の分野は特別な添 加物によって引き起こされた光学的性質を保有する新しいフォトニックス材料の合成に関するものである. 波長 可変の固体レーザーや非線型光学特性に関係する重要な結果やフォトクロミック材料について説明する.
\end{abstract}

[Received April 8, 1991]

The sol-gel technique offers a low temperature method for synthesizing amorphous inorganic solids which contain trapped organic molecules with deliberately chosen optical properties. The poor thermal stability of organic molecules has precluded their incorporation in traditional oxide host materials because of the elevated processing temperatures involved with these materials. Using the sol-gel approach, a wide variety of organic molecules have been incorporated in sol-gel matrices and demonstrated that they retain their specific optical properties in the sol-gel environment. Thus, it has been possible to use the sol-gel method to prepare photonic materials which are luminescent or photochromic, which possess nonlinear optical properties or which exhibit laser action. This paper reviews the two principal areas where sol-gel methods are used to produce organic-doped photonic materials. One area of activity involves the utilization of the organic dopants as luminescent probes of the sol-gel process. Examples of matrix rigidity and solvent chemistry changes are shown. The second area concerns the synthesis of new photonic materials whose optical properties are induced by the addition of specific dopants. Significant results involving tunable solid-state lasers, nonlinear optical properties and photochromic materials are presented.

Key-words : Sol-gel materials, Luminescence, Photonic materials, Lasers, Photochromics, Nonlinear optics, Chemical sensors

1. General aspects of luminescent sol-gel glasses

$7 \mathrm{HE}$ synthesis of glassy materials from gels has generated considerable interest in recent years. ${ }^{1)}$ The sol-gel process for making oxide glasses generally involves the use of metal alkoxides which undergo hydrolysis and condensation/polymerization reactions to give gels. ${ }^{2)-5}$ ) The process can ordinarily be divided into the following steps; forming a solution, gelation, drying and densification. The sol-gel method possesses a number of desirable characteristics. It enables one to prepare glasses at far lower temperatures than is possible by using conventional melting. In addition, it is a high purity process which leads to excellent homogeneity. Finally, the sol-gel approach is adaptable to producing bulk pieces as well as films and fibers.

During the mid-1980's several researchers recognized the potential for using the sol-gel process to incorporate organic molecules in an oxide matrix. ${ }^{6)-10)}$ That research represented the first step in the emergence of organic-doped gels as a novel approach for synthesizing photonic materials. Subsequently, research in this area has grown dramatically and today there are numerous examples of sol-gel materials which are luminescent, photochromic, possess nonlinear optical properties or exhibit laser action.

Luminescence has played a major role in the development of photonic sol-gel materials. The fact that the luminescence spectra for rhodamine $6 \mathrm{G}$ in silica sol-gels was changed only slightly from that of an alcohol solution ${ }^{6)}$ was the first indication that organic molecules were able to retain their spectroscopic properties in the gel host. The luminescent behavior thus indicated that the gel environment was conducive for producing optical properties as deter- 
mined by the excited state of the organic dopant. This feature is of direct importance for lasers, luminescent probes and nonlinear processes. In this section, we briefly review the materials systems, optical properties and chemical considerations for luminescent sol-gel materials.

There are two central features which have contributed to the development of luminescent sol-gels. First, there is the low processing temperatures involved in sol-gel synthesis. The ability to prepare an essentially inorganic oxide matrix with little or no heating means that the sol-gel process is thermally compatible with a wide variety of organic molecules. In contrast, the poor thermal stability of organic molecules precludes their incorporation in traditional oxide matrices. The second important feature is the ability to find solvents which are compatible with both the organic molecule and the sol-gel precursor. Although alcohol solutions are most frequently used, other solvents including NMP (N-methyl pyrrolidinone), DMF (dimethyl formamide) and THF (tetrahydrofuran) have widened the realm of organic molecules which can be incorporated in the gel matrix. Another consideration is the acid-base equilibria of the molecule. The $\mathrm{pH}$ of gels can vary substantially depending upon the type of matrix, the solvent and catalyst employed. As discussed below, the luminescent properties of certain dye molecules are sensitive to synthetic conditions primarily because of $\mathrm{pH}$.

The initial work on luminescent sol-gels emphasized the incorporation of laser dyes. Rhodamines, coumarins, oxazines and other well known dye molecules were investigated in a variety of hosts including $\mathrm{SiO}_{2}, \mathrm{SiO}_{2}-\mathrm{TiO}_{2}, \mathrm{Al}_{2} \mathrm{O}_{3}$ and composite solgels. ${ }^{6)-10)}$ In most cases, the organic molecule was dissolved either in the sol directly or in the same solvent as that used in the sol. In the composite sol-gels, the dye was dissolved in a monomer (e.g., methyl methacrylate) which was then impregnated in a porous silica gel and polymerized in-situ. ${ }^{9)}$ In addition to laser dyes, a large number of other luminescent organic molecules were also incorporated in solgel matrices. Several of these molecules (e.g., pyranine, pyrene) have been used as luminescent probes of the sol-gel process and are discussed in Part 2.

The initial work on luminescent sol-gels established the feasibility of using sol-gel methods to produce organic-doped photonic materials. The luminescence of these materials extends throughout the visible. Since the initial work, several new developments have occurred. Most notable has been the use of other oxide matrices including titanium and zirconium oxides, ${ }^{11)}$ phosphate gels ${ }^{12)}$ and organically modified oxides. ${ }^{13), 14)}$ In addition to several other organic and organometallic molecules being incorporated, metal clusters of $\mathrm{Mo}_{6} \mathrm{Cl}_{12}$ have also been used to prepare luminescent gels. ${ }^{15)}$

The work on luminescent gels has shown that these materials can possess a number of interesting spectroscopic features. Perhaps the most outstanding characteristic (discussed in Part 2) is the sensitivity of luminescent molecules to their environment. This feature has enabled the organic-doped gels to serve as luminescent probes of sol-gel chemistry and structure. Related to this has been the role of synthesis conditions in determining luminescent properties. Coumarin 4 exists in a variety of forms (cationic, zwitterionic, neutral, anionic), each of which exhibits specific emission bands. Experiments with silica gels doped with coumarin 4 have shown that the $\mathrm{pH}$ during sol synthesis determines the dominant luminescent form in the dried gel. ${ }^{16)}$ The results indicate that the $\mathrm{pH}$ of the sol determines the nature of the surface charges on pore walls which, in turn, determines the protonation of the coumarin dopant. Rhodamine B is also sensitive to acid-base equilibria, primarily through its carboxylic function. In this case, highly acidic conditions produce a lactonic form of rhodamine B which is colorless. ${ }^{17)}$ Another consideration with rhodamine dyes is dimer formation. There is evidence in silica gels that dimer formation is reduced and that dye molecules can be well isolated from interactions with neighboring molecules ${ }^{6)}$ Recent work indicates that dimerization of rhodamines is also suppressed in titanium oxide gels. ${ }^{11)}$

An area which is likely to receive increased attention in the future is that of energy transfer. Sol-gel materials provide an opportunity to investigate dyedye energy transfer as well as dye-ion energy transfer. In the latter case, the broad-band absorption of the dye should be particularly beneficial for efficient energy transfer. Early work by Avnir et al. indicated that dye-dye energy transfer was possible. ${ }^{7)}$ More recently, Genet and co-workers have demonstrated energy transfer in $\mathrm{ThPO}_{4}$ gels using an organic dye (coumarin 460) as the sensitizer and inorganic ions $\mathrm{Eu}^{3+}$ and $\mathrm{Tb}^{3+}$ as activators. ${ }^{22), 18)}$ It is not known if the process was radiative or non-radiative. In other related work, the presence of silver aggregates in the gel caused interaction to occur between rhodamine $6 \mathrm{G}$ and the silver aggregates and gave rise to increased absorption and emission from the dye. ${ }^{19}$ )

A fundamental part of characterizing the behavior of luminescent materials is to consider the inherent properties of the host. Silicate and aluminosilicate gels and xerogels are intrinsically luminescent, i.e., they luminesce in the blue region of the spectrum when irradiated with UV light even in the absence of a dopant molecule. This intrinsic luminescence must be taken into account when designing new photonic materials because it may obscure a desired property and/or because it may be mistaken for an induced optical property.

A representative spectrum, that of the intrinsic luminescence taken from a $\mathrm{SiO}_{2}$ xerogel prepared from $\mathrm{Si}\left(\mathrm{OC}_{2} \mathrm{H}_{5}\right)_{4}$ and excited at $350 \mathrm{~nm}$, is shown in 


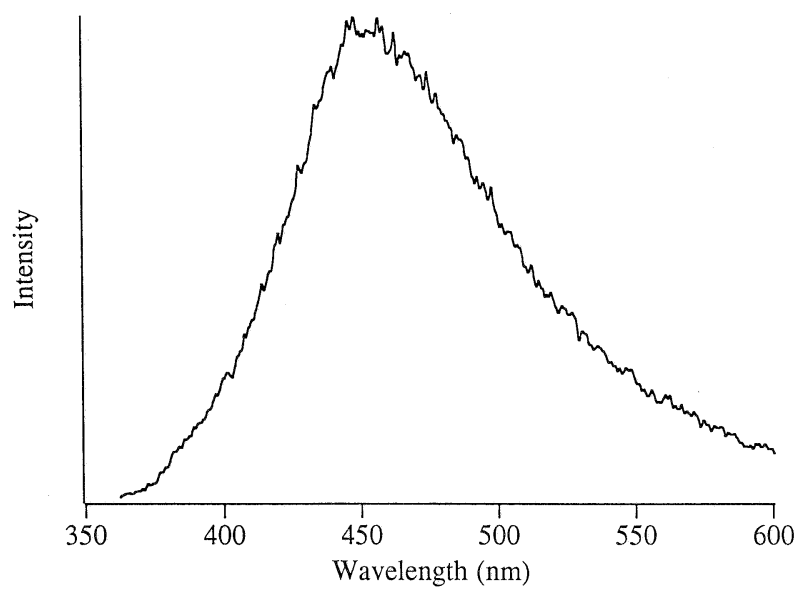

Fig. 1. Intrinsic luminescence from $\mathrm{SiO}_{2}$ xerogel prepared from TEOS. The excitation was at $351 \mathrm{~nm}$.

Fig. 1. The peak maximum occurs at $445 \mathrm{~nm}(22500$ $\mathrm{cm}^{-1}$ ) and the peak has a full width at half height of $95 \mathrm{~nm}\left(4550 \mathrm{~cm}^{-1}\right)$. The lifetime of the luminescence is on the order of $50 \mathrm{~ns}$. The intrinsic luminescence spectrum may vary slightly from sample to sample depending on the synthesis and processing conditions, but it always occurs in the blue region of the spectrum. This spectrum is similar to that observed from quartz and may be related to the E' defect center.

The most intense intrinsic luminescence occurs from xerogels. Sols do not luminesce at room temperature. The luminescence is first observed at the gel stage and the intensity increases as gelation and aging proceed. The intensity also increases as a function of decreasing temperature.

Aluminosilicate gels and xerogels also exhibit intrinsic luminescence although the intensity from these materials is significantly less than that from silicates. The spectrum is very similar to that shown in Fig. 1, and the luminescence also increases with decreasing temperatuure.

\section{Characterization of the gelation process}

The feasibility of using the sol-gel method to produce organic-doped glasses has led to two directions of study: use of dopant molecules as probes of the sol-gel process, and development of specific optical properties. In the following section we present the use of organic molecules as luminescent probes of the changes which occur during the processing route. In all of these studies, the spectroscopic response of the molecules to the desired environmental property (e.g., both structural and chemical) is first characterized and interpreted under controlled chemical and physical conditions. This information provides the basis for interpreting the spectroscopic response of the probe molecules in the sol-gel environment. Of the three probes discussed below, the results obtained with rigidochromism are empha- sized because of the unique insight this probe offers both for the sol-gel structure and rigidity at the microscopic level.

2.1 Rigidochromism as a probe of gelation, aging and drying ${ }^{20}$

The rigidochromic molecule bipyridyltriscarbonylchlororhenium(I), $\operatorname{ReCl}(\mathrm{CO})_{3}$ bipy, is a probe of the changes in rigidity of sols and gels prepared from tetraethoxysilane (TEOS) and diisobutoxyaluminotriethoxysilane (ASE). Rigidochromism, defined as the changes in the energy of the luminescence maximum as a function of the rigidity of the surrounding medium, ${ }^{21)}$ was measured in fluid and solid (frozen) reference solvents to calibrate the magnitude of the effect. The changes were then used to probe the rigidity of the two systems during gelation and drying. These studies revealed large differences between the two systems.

The behavior of the emission maximum of $\mathrm{ReCl}(\mathrm{CO})_{3}$ bipy as a function of temperature in liquid TEOS before gelation is shown in Fig. 2. This behavior is similar to that observed in ethanol. Above the freezing point of the solution, the emission maximum is $605 \mathrm{~nm}\left(16530 \mathrm{~cm}^{-1}\right)$. As the temperature is decreased, the emission maximum is virtually unchanged down to a temperature of about $180 \mathrm{~K}$. A sudden blue shift is observed in the region of the freezing point of the mixture. The band maximum in the frozen solution is about $530 \mathrm{~nm}\left(18870 \mathrm{~cm}^{-1}\right)$.

The emission spectra of $\mathrm{ReCl}(\mathrm{CO})_{3}$ bipy in the dried gel formed from TEOS were studied for comparison with the fluid solution/frozen solution data. The position of the band maximum as a function of temperature in this medium is shown in Fig. 2. The position of the band maximum is independent of temperature over the range studied. It coincides with the position of the band maximum in the solid frozen solution of TEOS within experimental error.

Luminescence spectroscopy in the aluminosilicate and TEOS sols and gels

The band maximum of the emission of

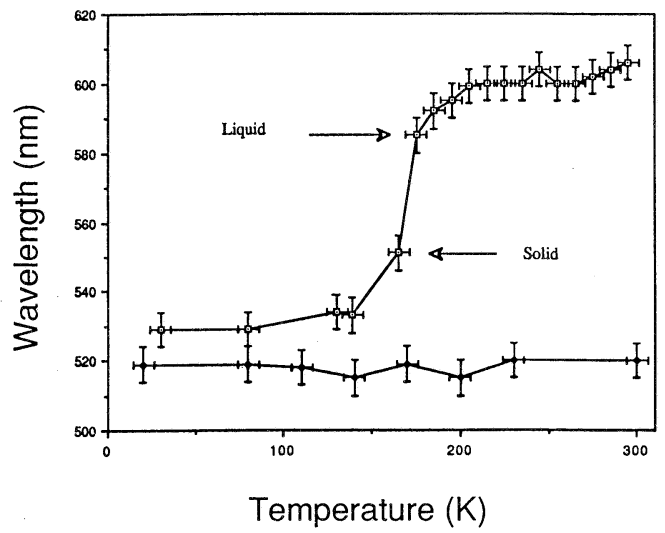

Fig. 2. Plot of the wavelength of the emission maxima (nm) of $\operatorname{ReCl}(\mathrm{CO})_{3}$ bipy as a function of temperature in TEOS sol (top) and in the rigid gel (bottom). 
$\mathrm{ReCl}(\mathrm{CO})_{3}$ bipy in ASE exhibits large shifts during the gelation and drying processes. The emission band in the liquid state just after the mixing of the components is broad and centered around $600 \mathrm{~nm}$ $\left(v=16660 \mathrm{~cm}^{-1}, \Delta v_{1 / 2}=2500 \mathrm{~cm}^{-1}\right)$. No change is observed at the gelation point although the solution has turned to a solid amorphous material which does not flow when the container is tilted. After a long period of aging in a closed container, the emission maximum is blue shifted to $560 \mathrm{~nm}\left(v=17860 \mathrm{~cm}^{-1}\right.$, $\left.\Delta v_{1 / 2}=3650 \mathrm{~cm}^{-1}\right)$. At the end of the drying, the emission peak has shifted to $523 \mathrm{~nm} \quad(v=19120$ $\mathrm{cm}^{-1}$ ) and has a width of about $3580 \mathrm{~cm}^{-1}$. The small change in the FWHM indicates that the position of the band maximum has uniformly shifted and that the effect is not caused by the growth of a new band with a concomitant decline of the original band.

The shifts of the position of the band maximum and changes in the weight of the sample as a function of time are shown in Fig. 3. For convenience, the processing time is plotted on a logarithmic scale. The initial evolution of $\mathrm{ReCl}(\mathrm{CO})_{3}$ bipy emission as a function of time consists of a continuous shift of the luminescence maximum to $560 \mathrm{~nm}$. No loss of solvent occurs during this time period. Surprisingly, the shift does not occur during the sol gel transition but instead begins at a later time during the aging of the gels. After a time span of about 5 to 6 times the gelation time, the shift of the emission maximum ceases. The next step is clearly related to the drying of the gel. The shift of the emission maximum occurs continuously with the decrease in weight of the sample due to removal of solvents (initially representing nearly $80 \%$ in volume of the gel). The linear shrinkage $(\Delta l / l)$ of the gel after drying is about $50 \%$ and causes an eightfold increase in the concentration of $\mathrm{ReCl}(\mathrm{CO})_{3}$ bipy.

Exactly the same procedure is followed for the preparation of silica sonogels containing the rigidochromic probe. However, the TEOS system doped with $\mathrm{ReCl}(\mathrm{CO})_{3}$ bipy exhibits a very different behavior. From the initial liquid state to almost the

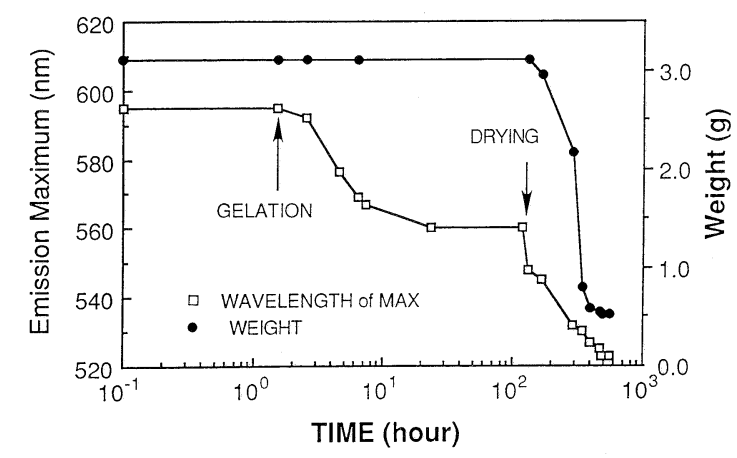

Fig. 3. Plot of the wavelength of the emission maxima of $\mathrm{ReCl}(\mathrm{CO})_{3}$ bipy in the aluminosilicate system as a function of processing time (lower curve). The concomitant weight changes are shown in the upper curve. end of drying, the emission maximum remains fixed at about $600 \mathrm{~nm}$. A blue shift only occurs with the final removal of the alcohol.

\section{$\mathrm{ReCl}(\mathrm{CO})_{3}$ bipy as a probe of gel rigidity}

The evolution of the $\mathrm{ReCl}(\mathrm{CO})_{3}$ bipy luminescence shown in Fig. 3 can be separated into three distinct parts, each of them related to a specific sequential step of the sol gel transformation: gelation, aging, and drying. First, during the gelation stage, the most drastic chemical and physical changes occur. The initial sol, which is completely fluid, is transformed into a solid and brittle material. After the addition of water, small particles on the order of 10-20 $\AA$ in diameter are rapidly formed as a consequence of the hydrolysis of the Al-OR groups to $\mathrm{Al}-\mathrm{OH}$ and the condensation of the $\mathrm{Al}-\mathrm{OH}$ groups into - $\mathrm{Al}-\mathrm{O}-$ Al- polymers. After this step, a slow aggregation process takes place which forms inorganic clusters of increasing size. These clusters have been characterized and have a ramified and open structure. When a critical cluster size is reached the viscosity of the sols sharply increases and the solution becomes rigid. ${ }^{22)}$ Interestingly, the $\mathrm{ReCl}(\mathrm{CO})_{3}$ bipy probe molecule is insensitive to these changes and the emission peak maximum remains constant at $590 \mathrm{~nm}$. This observation indicates that although the gel is macroscopically solid, the rigidochromic molecules are unconstrained in a solvent-like environment. The solvent phase at the gel point consists of propanol, water (i.e., the water not used for hydrolysis or released by condensation reactions) and the additional ethanol and butanol produced by the hydrolysis of the alkoxy groups. This phase constitutes the largest volume fraction of the gel. The solid-phase oxide network which causes the observed macroscopic rigidity only represents a few percent of the total gel volume. At this stage, the mobility of the $\mathrm{ReCl}(\mathrm{CO})_{3}$ bipy molecules is not constrained by the gel structure. Subsequently, the luminescence is the same as in the liquid sol.

The second step of the process, the aging period, takes place in a sealed vessel. Although the gel is kept in a closed container and no evaporation of the organic molecules occurs, the luminescence maximum continuously blue shifts. At the end of the aging period, the emission maximum stabilizes at 560 $\mathrm{nm}$, intermediate between the value observed in a completely fluid medium, $600 \mathrm{~nm}$, and that in a completely solid medium, $525 \mathrm{~nm}$. The blue shift during this period is caused by partial rigidification of the gel on the molecular level. It indicates that the $\mathrm{ReCl}(\mathrm{CO})_{3}$ bipy probe molecules are sensitive to structural changes in the aluminosilicate gel. The rigidochromic probe is not simply surrounded by the interstitial liquid phase as it was during the gelation stage, but now it becomes somewhat entrapped in the oxide polymer network. At the gel point, the gel network is a very ramified and open structure which does not restrict the mobility of the rigidochromic 
molecules. The evolution of the fluorescence during the aging period shows that the motion of $\mathrm{ReCl}(\mathrm{CO})_{3}$ bipy molecules is progressively restricted. These results indicate that large modifications in the local gel structure occur during the aging period which increase the rigidity of the oxide skeleton. These structural modifications are in agreement with the reported chemical changes in the surface of the oxide network of the aluminosilicate probed by the luminescence of pyranine. ${ }^{23)}$ Condensation reactions between $\mathrm{Al}-\mathrm{OH}$ groups are probably responsible for the local evolution of the polymers which leads to a more compact network and increases the gel rigidity during the aging process. One macroscopically observable property which is indicative of these changes is the gel hardness, which increases by a factor of five during the same period of time. ${ }^{24)}$

The third stage of the process, which is related to the final changes of the emission of $\mathrm{ReCl}(\mathrm{CO})_{3}$ bipy, is the drying of the gel. When the solvents are removed, the gel structure collapses and the gel shrinks continuously. The final volume is about one eighth of that of the aged gel. As indicated by the blue shift of the emission of $\operatorname{ReCl}(\mathrm{CO})_{3}$ bipy, this step is accompanied by a progressive and eventually complete rigidification of the matrix. The final emission wavelength is the same as that in frozen ethanol solution. Both the increase in surface energy due to modification of the interface between the oxide polymer and the liquid and the capillary forces of the solvents provide the driving force for this partial densification. New condensation reactions occur because some reactive groups are in closer proximity. As a result, the oxide network becomes more compact. Its flexibility drastically decreases with the departure of the solvating species. The $\mathrm{ReCl}(\mathrm{CO})_{3}$ bipy molecules, which were previously entrapped in the network, are now completely immobilized by this collapsing structure. Moreover, the probe molecules can not be leached from the gel. It is usually during the drying process that gels develop cracks because of the increasing rigidity and by the capillary force-induced strains that can not be released by changes in the gel shape and morphology.

\section{Behavior of TEOS gels}

The rigidochromic probe molecule was also used to study the gelation, aging and drying of the TEOS system. The probe showed quite different behavior compared to that in the aluminosilicate system. The wavelength of the emission maximum remains constant at $590 \mathrm{~nm}$ from the initial liquid state through the gelation process to almost the end of the drying. The blue shift indicative of rigidity occurred only with the final removal of the organic molecules. This contrasting behavior of the silica sonogel indicates that there are significant structural differences between the two kinds of investigated systems and that $\operatorname{ReCl}(\mathrm{CO})_{3}$ bipy is a sensitive probe of this differ- ence. In the silica sonogels, the constancy of the emission at $590 \mathrm{~nm}$ indicates that the probe molecules are in a non-rigid environment during gelation, aging and a major period of the drying. The fact that this behavior is different from that of aluminosilicate gels is not surprising. Indeed,the chemistry of the precursors, the mechanisms of polymerization and, therefore, the structure of the gels are different. ${ }^{25}$ ) Moreover, the characteristics of the pore walls including polarity and electric charge are expected to be substantially different. This may change the interactions of the dye with the oxide network and consequently its position inside the gel structure. In the TEOS derived gel, the blue shift only occurs with the final loss of solvents, suggesting that during gelation and aging the probe molecules are confined in the interstitial liquid phase and are not incorporated into the silica polymer network. The adsorption of the active dyes on the surface of a pore once the water and ethanol are almost completely evaporated may account for the evolution of the $\operatorname{ReCl}(\mathrm{CO})_{3}$ bipy fluorescence towards the characteristic blue shift of the rigidified state. In the TEOS system, it is impossible to exactly follow the final shift of the maximum on the emission spectrum of $\mathrm{ReCl}(\mathrm{CO})_{3}$ bipy incorporated into the rigid system because of the overlap of the luminescence peak with an intense peak from the intrinsic emission of the gel. It is clear, however, that the peak has shifted to about $530 \mathrm{~nm}$, indicative of a rigid system.

2.2 Pyranine as a probe of chemical changes ${ }^{23)}$

A second important aspect of the gelation process which has been studied by using molecular probes is the chemical change which occurs during the polymerization reactions. Of particular interest to us were the changes in the water/alcohol ratio during gelation. As the hydrolysis reaction occurs, alcohol is continuously being produced and the ratio of the amount of water to the amount of alcohol should continuously decrease. It is desirable to probe these changes on the molecular level. The fluorescence spectrum of pyranine, 8-hydroxy 1, 3, 6-trisulfonated pyrene, is sensitive to proton-transfer phenomena. ${ }^{26)}$ The molecule can be encapsulated in gel-glasses and retain its fluorescence properties in the materials. As discussed below, the fluorescence spectrum is sensitive to the proton acceptor ability of the medium surrounding the molecule and can be used to monitor changes in the water/alcohol ratio.

The spectroscopy of pyranine was studied in control solutions consisting of water/propanol mixtures. The protonated form of the molecule emits in the blue region of the spectrum at $430 \mathrm{~nm}$ whereas the deprotonated form emits in the green at $515 \mathrm{~nm}$. The relative intensities of the blue and green emission bands depend on the surrounding medium. Emission spectra of pyranine in test solutions are shown in Fig. 4. In water rich environments the green emission band dominates because water acts 


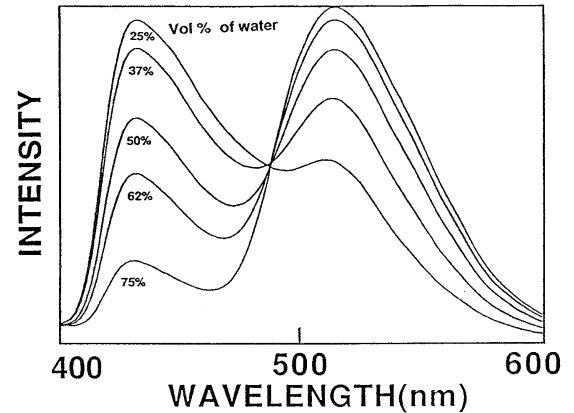

Fig. 4. Emission spectrum of pyranine dissolved in water/ propanol reference solutions (vol\% water is indicated). The excitation is at $345 \mathrm{~nm}$.

as a proton acceptor. In propanol rich environments, the blue band is dominant because the pyranine remains protonated. The ratio of the intensities, called " $F$ " in our work, is defined as the intensity of the blue peak divided by that of the green peak. A more rigorous determination of $F$, which involves normalizing the two spectra and subtracting the pure green emission curve, did not lead to significant differences from that of the simple ratio.

The changes in the ratio, $F$, which accompany aging and drying of the pyranine-doped aluminosilicate gels are shown in Fig. 5. Consider first the initial part of the figure up to a processing time of about 150 hours. The ratio $F$ increases smoothly during the gelation process from $t=0$ to an intermediate value of $F=0.45$ at $t=15$ hours at the gel point. The ratio continues to evolve in closed gels over a period of time equal to about 8 times the gelation time, eventually reaching a maximum value of $F=0.9$ after 200 hours. These results indicate that changes in the gel chemistry continue well beyond the gelation period. The macroscopic rigidity observed at the gel point does not mean that the reactive entities are immobile at the microscopic level. The rates of the reactions involved in the aging of the gel appear to be slower than those in the liquid state, but the extent of these changes is as great in the gel state as in the liquid state.

Drying experiments were carried out after the lu-

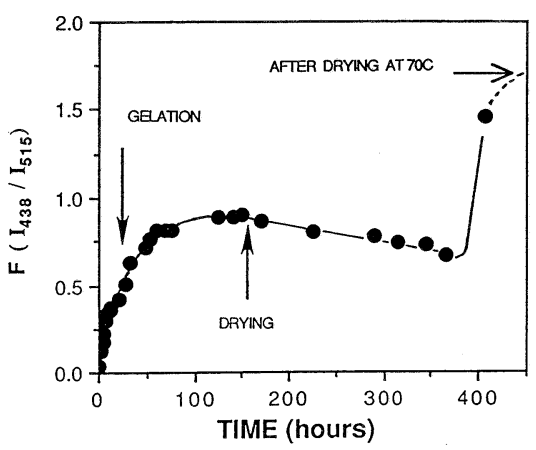

Fig. 5. Evolution of the emission intensity ratio $(F)$ during gelation, aging and drying processes. minescence of the sealed gel reached a stable value. The ratio $F$ decreased slowly from 0.9 to 0.7 during the first $60 \%$ of weight loss. In contrast, the curve becomes irregular when the weight loss reaches $70 \%$. At this stage $F$ increases sharply to a maximum of 1.6. This value is comparable to that observed for a gel that has been heated for 10 hours under vacuum at $70^{\circ} \mathrm{C}$ and is well dried.

The spectral changes which occur are significant despite the fact that aluminosilicate sols do not undergo significant compositional changes. These results suggest that the pyranine molecules are probing a very selected aspect of the gel polymerization chemistry which is strongly influenced by the protonation/deprotonation characteristics of the solvation shell. The initial value of $F$ just after hydrolysis is substantially different from that in a water/propanol mixture with the same water concentration. In the early moments of the gelation process, pyranine is not homogeneously distributed in the solution. Rather, it appears that the probe is already part of the small particles which result from partial condensation of the $\mathrm{Al}-\mathrm{OH}$ groups. These particles, which have both water and pyranine molecules adsorbed, constitute a medium with a substantially higher proton donor ability than the average composition of the sol.

The continuous changes in the ratio $F$ during gelation and aging indicate that significant chemical modifications occur both in the adsorbed layers and consequently in the solvation sphere of the pyranine molecules. The increase in $F$ from 0 just after the solution preparation to 0.45 at the gel point and eventually to 0.9 at the end of aging indicates that the proton acceptor ability of the adsorbed layers on the polymer surface decreases substantially. Ultimately an equilibrium is established which reflects the initial composition of the sol. The changes in emission during the drying of the gels can be explained on a similar basis. Propanol is the most abundant solvent present in the pore system of the gel and has a higher vapor pressure than water. The first step of drying corresponds to the vaporization of propanol which gives rise to an increase in the relative concentration of water in the remaining solvent. The ratio $F$ decreases accordingly. Later as drying continues, the free water content approaches zero and the ratio $F$ increases sharply.

In must be emphasized that the above results are specific to aluminosilicate gels. The results contrast with those obtained from silica gels because large differences exist due to different polarities, polymerization mechanisms and oxide network structures. ${ }^{27}$ ).

An important discovery made with the pyranine system has opened up an entirely new application for doped sol-gel glasses. The pyranine molecules which are trapped in the oxide network are found to be sensitive to an external solvent environment! Aged gels were immersed in water or propanol. The lumines- 
cence changes from green, when water is used for infiltration, to predominantly blue when propanol is diffused into the gels. This effect is reversible, and no leaching of the dyes occurs during the solvent exchange. This discovery was a definitive indication that doped gel-glasses can be used as optically based chemical sensors. The potential of this application is discussed in Part 3.1.

\subsection{Pyrene}

Chemical and physical changes which occur during the sol-gel-xerogel transitions have been studied by using pyrene as a photophysical probe. ${ }^{28)}$ The luminescence spectrum of this molecule exhibits vibronic structure. The ratio of the intensities of the first to fifth peaks in the spectrum $\left(I_{1} / I_{5}\right)$ is sensitive to the polarity of the environment. The ratio increases with increase in polarity. In addition, pyrene forms excimers, i.e., complexes between one pyrene in its lowest excited state and a second pyrene in its ground state. The intensity of the excimer emission increases with increase in the concentration of the molecule and with trapping in small pores. Both of these changes facilitate the intermolecular complexation.

Changes in the emission spectra of pyrene and of the excimer of pyrene were followed as a function of gelation time. The following conclusions were reached. On a molecular scale, geometric complexity and irregularity start to build up mainly after the gelation point. Two general patterns of behavior were observed depending on the water to silane ratio. At low ratios, geometric irregularity and porosity build up gradually along the whole process, resulting first in an increase in pyrene emission intensity as aggregation became more pronounced, followed by a decrease in intensity as pyrene molecules became isolated. At high water to silane ratios, a smooth surface was formed. These results suggested that polymerization-gelation occurs at low water to silane ratios whereas formation of a colloid followed by its gelation occurs at high water to silane ratios. Ethoxy groups slow down the polymerization in comparison with methoxy groups, but the reaction rates coincide once all of the alkoxy groups are hydrolyzed. The pyrene excimer disappears at the final xerogel state, proving that the sol-gel process is an effective method for isolating organic molecules. The changes in the polarity of the pyrene environment along the sol-gel process, as reflected by the $I_{1} / I_{5}$ ratio, are due to pyrene aggregation and not due to the support itself.

The fluorescence of pyrene probe molecules has also been used to follow the formation of micelles in sol-gel glasses. ${ }^{29)}$ The vibronic structure intensity ratio was different in the sol, gel and xerogel stages. When the concentration of sodium dodecyl sulfate, the micelle former, was high, the ratio had values similar to those of solutions of micelles. This result suggested that micelles are trapped in the sol-gel glasses.

\section{New optical materials}

The synthesis of new photonic materials is the second of the two directions stimulated by the ability of the sol-gel method to incorporate organics. In this case, we induce the optical properties of the gel based upon the deliberately chosen dopant. For example, when the desired photonic material is to be a new solid state gain medium, laser dyes of the desired wavelength region are added. Other examples include the addition of soluble organic polymers and molecules to cause nonlinear optical effects, indicator molecules to produce optically-based chemical sensors and photochromic molecules to produce optical memory and switching. In the following section we review progress in these areas with particular emphasis on recent results from our laboratories.

\subsection{Sol-gel lasers}

There has long been an interest in fabricating solid state gain media containing organic laser dyes. Organic laser dyes possess several advantages over their inorganic counterparts. Their cross-sections for absorption and emission are orders of magnitude greater than lanthanide or transition metal ions and they exhibit lower threshold powers for laser action. Their gain characteristics and tunability in the visible spectrum are also quite attractive for device applications. Despite the advantages offered by solidstate gain media, attempts to incorporate organic dye materials in polymeric hosts have met with limited success. For example, rhodamine 6G (R6G) incorporated in a mixture of methyl methacrylate and (poly) methyl methacrylate (PMMA) was reported to have a laser efficiency of $36 \%$, but lacked photostability. ${ }^{30)}$ In general, polymeric hosts have not exhibited the necessary thermal and mechanical properties, photostability or refractive index uniformity to become successful laser hosts. In contrast, inorganic glasses offer superior optical, thermal and chemical properties. The problem here is that the organic dyes cannot withstand the thermal conditions posed by the processing temperatures of conventional glasses.

The sol-gel technique offers a low temperature method for synthesizing amorphous materials which are essentially inorganic. The initial work on preparing dye-doped sol-gel glasses strongly suggested that solid-state tunable lasers based on sol-gel matrices were feasible. Both the alkoxide precursors and the common laser dye families (coumarins, xanthenes, oxazines) are soluble in the same solvents ensuring that the dyes could be incorporated in the matrix without undesirable aggregation effects. A particularly attractive feature of these materials is that by providing the dye with an inorganic environment, increased photostability is possible.

Research activities during the past three years 
Table 1. Summary of sol-gel laser systems.

\begin{tabular}{|c|c|c|c|c|}
\hline Dye & Matrix & $\begin{array}{l}\text { Oscillating Range (peak) } \\
\text { in } \mathrm{nm}\end{array}$ & $\begin{array}{c}\text { pump source } \\
\text { (wavelength in } \mathrm{nm} \text { ) } \\
\end{array}$ & ref. \\
\hline Coumarin 1 & $\mathrm{SiO}_{2} * * *$ & $433-457(444)$ & $\mathrm{XeCl}$ Excimer (308nm) & 16 \\
\hline Coumarin 102 & $\mathrm{SiO}_{2}^{* * *}$ & $487-495(491)$ & $\mathrm{XeCl}$ Excimer (308nm) & 16 \\
\hline Coumarin 153 & $\mathrm{SiO}_{2}^{* * *}$ & $545-572(558)$ & $\mathrm{XeCl}$ Excimer (308nm) & 16 \\
\hline Rhodamine B & $\mathrm{SiO}_{2}$ & $612-620(618)$ & $\mathrm{XeCl}$ Excimer (308nm) & 46 \\
\hline Sulforhodamine 640 & $\mathrm{SiO}_{2}$ & $605-647(621)$ & Dbl Nd:YAG (532nm) & 34 \\
\hline Rhodamine $6 \mathrm{G}$ & ASE & $561-580(569)$ & Dye Laser (540nm) & 36 \\
\hline Coumarin 153 & ASE & $545-565(555)$ & Dye Laser $(460 \mathrm{~nm})$ & 44 \\
\hline Fluorescein & ASE & $545-559(552)$ & Dye Laser $(511 \mathrm{~nm})$ & 44 \\
\hline BASF 241 (perylene derivative) & MMA impregnated $\mathrm{SiO}_{2}$ & $568-583(575)$ & Dbl Nd:YAG (532nm) & 37 \\
\hline Rhodamine $6 \mathrm{G}$ & ORMOSIL (EP/MA) & $557-598(568)$ & Dye Laser (540nm) & 14 \\
\hline Rhodamine $6 \mathrm{G}$ & ORMOSIL (EP) & $559-587(571)$ & Dye Laser (540nm) & 14 \\
\hline Coumarin 153 & ORMOSIL (MA) & $498-574(525)$ & Dye Laser $(460 \mathrm{~nm})$ & 14 \\
\hline Rhodamine $6 \mathrm{G}$ & ORMOSIL (MA) & $562-590(570)$ & Dbl Nd:YAG (532nm) & 39 \\
\hline Sulforhodamine B & ORMOSIL (MA) & $600-625(609)$ & Dbl Nd:YAG (532nm) & 42 \\
\hline Rhodamine B & ORMOSIL (MA) & $593-607(598)$ & Dbl Nd:YAG (532nm) & 42 \\
\hline Nile Blue & ORMOSIL (MA) & $680-746(696)$ & Dbl Nd:YAG (532nm) & 42 \\
\hline
\end{tabular}

have clearly established that tunable solid state lasers based on sol-gel glasses can be achieved. A variety of laser dyes doped in different sol-gel matrices have demonstrated laser action. The initial report involving dye-doped sol-gels was performed using aged silica and sodium silicate gels. ${ }^{31)}$ The laser threshold and conversion efficiency for rhodamine 6G (R6G) doped systems were reported to be comparable to an ethanol solution of the dye. Since that time, a substantial amount of work has been carried out on solid-state systems. To date, different rhodamine, coumarin and perylene dyes have lased in a variety of matrices including silica, alumina, aluminosilicate, organic modified silicates (ORMOSILs) and sol-gel composites. ${ }^{14), 16), 32)-36)} \mathrm{A}$ summary of solid-state sol-gel lasers, their dyes and wavelengths over which lasing occurs is provided in Table 1.

Specific laser characteristics have been explored in relatively few systems and only initial results have been reported. The most complete work is available for sulforhodamine B (SRB) in dried silica gel, perylene in a sol-gel composite which contained methylmethacrylate as the polymer phase, and for coumarin and rhodamine doped ORMOSILs. Tunability has been demonstrated in the SRB/silica system $(600 \mathrm{~nm}$ to $650 \mathrm{~nm}),{ }^{34)}$ the perylene/composite system $\left(568 \mathrm{~nm}\right.$ to $583 \mathrm{~nm}$ ) ${ }^{37}$ ) and in SRB/ORMOSIL $(570 \mathrm{~nm}$ to $590 \mathrm{~nm}) .{ }^{38)}$ The threshold for lasing has been reported for these systems and is in the range of 1 to $10 \mu \mathrm{J}$, although one must be aware of the pump source for each of these measurements. Slope efficiencies show substantial variation among the systems; from $8 \%$ for the perylene/composite to nearly $40 \%$ for SRB/ORMOSIL. ${ }^{39)}$ The question of slope efficiency may be very dependent upon the host matrix and is discussed below in greater detail.

Optical gain measurements have been reported for aged silica gels (wet gels) and for (solid) ORMOSIL

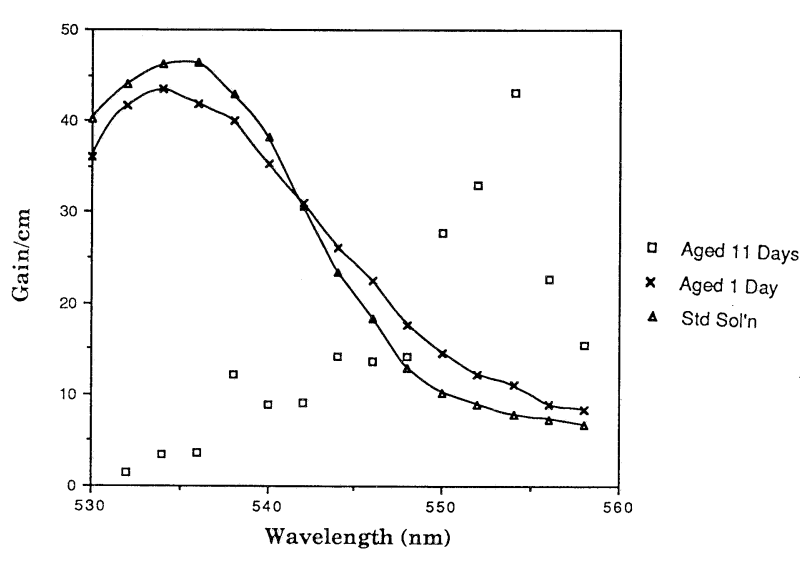

Fig. 6. Spectral gain envelope for coumarin 153 in ethanol and in aged (silica) gels.

samples. ${ }^{14), 16)}$ Spectral gain envelopes for coumarin 153 in aged silica gels and in an ethanol reference solution are shown in Fig. 6 . The gel which was aged for one day is quite similar to that of the ethanol reference. After eleven days of aging, however, the spectrum has changed significantly. A sizeable 19 $\mathrm{nm}$ red shift has occurred in the peak of the gain curve. The red shift is consistent with what is expected from the local chemical changes which are known to occur during the aging of silica gels. During the aging process, condensation of the hydrolyzed species produces additional water which enters the solvent phase. Jones et al. have shown that coumarin dyes in ethanol-water solutions are red shifted substantially from those of pure ethanol. ${ }^{40)}$ It is important to note that aging has caused very little reduction in the peak gain and thus it is possible to obtain high values of optical gain in a well aged gel material. A similar series of measurements have been performed with rhodamine doped silica gels. The optical gain characteristics are similar, although the susceptibility of R6G to dimerization effectively reduces the gain 
Table 2. Spectral gain characteristics of dye-doped gels.

$\begin{array}{clcc}\text { Dye } & \text { Medium } & \text { Max. Gain }\left(\mathrm{cm}^{-1}\right) & \text { Peak Wavelength (nm) } \\ \text { Coumarin 153 } & & & \\ & \text { EtOH soln } & 47 & 535 \\ \left(3.4 \times 10^{-3} \mathrm{~mol} / \mathrm{l}\right) & \text { Aged } 1 \text { day } & 43 & 534 \\ & \text { Aged } 4 \text { days } & 40 & 550 \\ & \text { Aged } 11 \text { days } & 41 & 554 \\ & \text { Ormosil (solid) } & 40 & 537\end{array}$

$\begin{array}{llll}\text { Rhodamine } 6 \mathrm{G} & & \\ \left(4.0 \times 10^{-3} \mathrm{~mol} / \mathrm{)}\right) & \text { EtOH soln } & 45 & 585 \\ & \text { Aged } 1 \text { day } & 40 & 585 \\ & \text { Aged } 4 \text { days } & 31 & 587 \\ & \text { Aged 11 days } & 10 & 590\end{array}$

values during the aging process. Table 2 summarizes the spectral gain characteristics for coumarin 153 and R6G in aged silica gels.

Optical gain measurements on dried silica gels are problematic because shrinkage during the drying stage produces poor optical quality surfaces. Polishing of dried gels does not lead to a significant improvement in optical quality. In contrast, ORMOSIL samples can be polished by standard methods and are able to achieve optical quality finishes on solid surfaces. The optical gain values determined for coumarin 153/ORMOSIL samples were found to be virtually identical to those of the ethanol reference, ${ }^{14)}$ underscoring the promise of these materials for tunable laser applications. From the few gain measurements reported to date and the corresponding high values obtained, it is evident why laser action is readily achieved in these systems using rather modest laser cavities.

The role of the sol-gel matrix is likely to be a critical question in the development of dye-doped sol-gel lasers. A central issue to be considered is how the sol-gel matrix influences such laser characteristics as conversion efficiency, photodegradation behavior and laser lifetime, and how these properties compare to those of polymeric hosts. In the relatively few results available, it is evident that conversion efficiencies of rhodamine doped sol-gel materials are substantially dependent upon the matrix. Values range from $2 \%$ for R6G in alumina films ${ }^{32}$ ) through approximately $25 \%$ for R6G in aluminosilicate xerogels, ${ }^{36}$ ) $30 \%$ in R6G/ORMOSIL ${ }^{39)}$ to $40 \%$ in aged silica gels. ${ }^{31)}$ In the case of sulforhodamine B (SRB), the slope efficiency for SRB/ORMOSIL was $39 \%,{ }^{39)}$ which is nearly twice as large as that reported for SRB in dried silica gel. ${ }^{34)}$ The pumping conditions for these two SRB systems were quite comparable, although the ORMOSIL employed a considerably greater pump energy $(600 \mu \mathrm{J})$.

Matrix effects are also apparent in the laser emission bands for rhodamine doped sol-gels. Table 1 summarizes the matrix effects for SRB and R6G. Other results with ORMOSILs demonstrate that laser emission spectra are definitely affected by the constituent present in the matrix. ORMOSIL gels modified by epoxy-diol exhibit different characteristics depending upon the presence of methyl methacrylate (MMA) as a copolymer. Figure 7 illustrates that both the emission peak and the oscillation band are influenced by the presence of MMA; the presence of MMA causes a blue shift in the laser peak and produces a substantially wider oscillation band. In contrast to the laser characteristics, the fluorescence emission spectra are unaffected by the presence of MMA.

Photostability represents one of the most important issues for sol-gel laser materials. Photodegradation under laser action provides a rigorous assessment of this behavior. R6G in alumina films exhibited a $50 \%$ output reduction in laser intensity within 600 pulses when pumped at $1 \mathrm{MW} / \mathrm{cm}^{2}{ }^{32}$ R6G in ASE monoliths pumped at $1 \mathrm{~mJ} /$ pulse reached the $50 \%$ level in 1500 pulses $(1 \mathrm{~Hz}$ repetition rate) and continued to exhibit laser action after 40,000 pulses. ${ }^{36)}$ No specific results were reported for SRB/ silica, however, there was an indication that photostability was relatively poor as thermal effects at low pumping rates $(10 \mathrm{~Hz})$ led to decreased output power. ${ }^{34)}$ In the perylene/composite system, encouraging results were mentioned as there was apparently no change in laser performance after "several thousand" pulses, but no details of these experi-
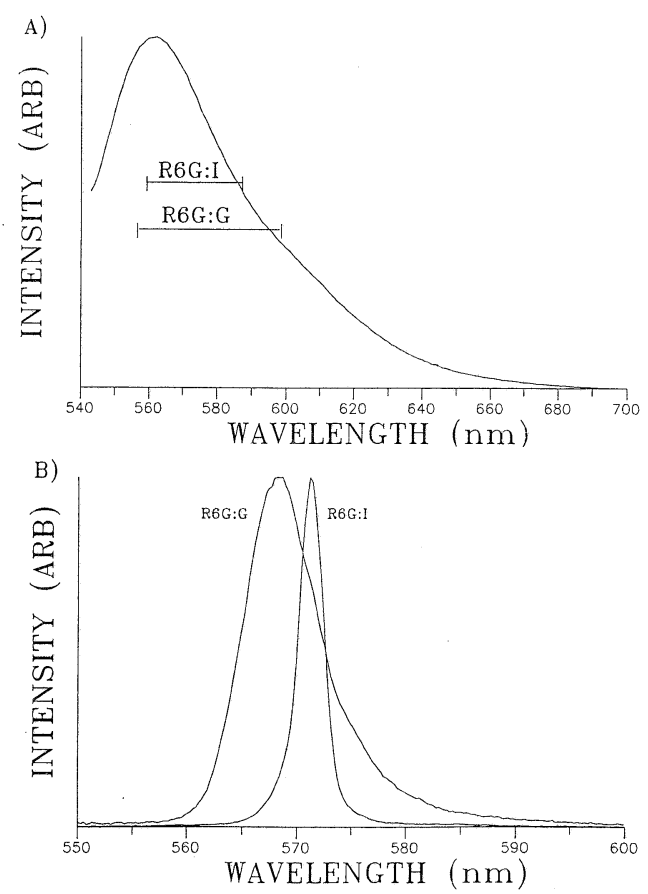

Fig. 7. Upper curve: Emission spectrum for rhodamine 6Gdoped ORMOSIL excited at $539 \mathrm{~nm}$. Laser oscillation ranges are superimposed for the two R6G materials in the lower curve. Lower curve: Free running laser oscillation for R6G in two ORMOSIL matrices. Matrix I is an epoxy-diol silicate; matrix $\mathrm{G}$ is a similar epoxy-diol silicate containing methyl methacrylate. 
ments were provided. ${ }^{41)}$

The photostability of dye-doped ORMOSILs have been studied in greater detail. ${ }^{39), 42)}$ Recent work has shown that these materials possess substantially longer lifetimes and operate at much higher repetition rates than the other sol-gel systems or polymer hosts. Rhodamine $6 \mathrm{G}$ and sulforhodamine B doped ORMOSILs reach the 50\% laser output level after 11,000 and 14,500 pulses, respectively, when pumped at $95 \mu \mathrm{J}(8 \mathrm{~kW})$ at $30 \mathrm{~Hz}$. In addition, initial experiments indicate that doubling the pump energy to $180 \mu \mathrm{J}(15 \mathrm{~kW})$ has a relatively small effect; the $50 \%$ level is reached in 12,000 pulses. The lifetime characteristics (Fig. 8) generally follow a double exponential decay rate. There is an initial region (3600 pulses) where the laser output decreases a modest $10 \%$ for all samples. Thereafter, the rates increase. In general, the ORMOSILs doped with SRB have a longer lifetime than those doped with R6G; this behavior has been attributed to the better match of the excitation spectrum to the $532 \mathrm{~nm}$ pump source. ${ }^{42)}$ The results in Fig. 8 also illustrate that the lifetime is affected by the pulse repetition rate. As the rate increases from 6 to $30 \mathrm{~Hz}$, the $50 \%$ laser output level varies from 18,500 to 11,000 pulses.

\subsection{Nonlinear optical materials}

The promise of photonics technology to transmit, process and store information with enormous improvements in speed, band width and storage density has stimulated considerable research over the past decade. There continues to be a vital need for new materials whose $\chi^{(2)}$ and $\chi^{(3)}$ (the second and third order nonlinear susceptibilities, respectively) can be used to produce the various nonlinear functions necessary for such operations as optical information

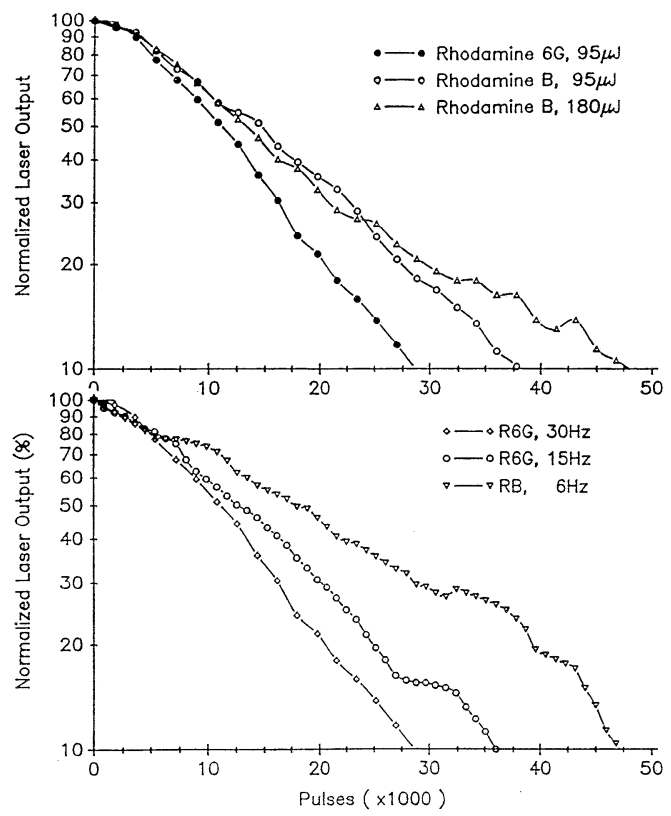

Fig. 8. Laser lifetimes for rhodamine-doped ORMOSILs pumped at $532 \mathrm{~nm}$. Upper curve: Dependence on pump energy. Lower curve: Dependence on pump repetition rate. processing and optical data storage. In general, the desired nonlinear material must not only possess large $\chi^{(2)}$ and $\chi^{(3)}$ but also exhibit good chemical and mechanical stability, low optical loss, a high optical damage threshold and be fabricable into useful forms such as fibers and films. The application of the sol-gel method to synthesize nonlinear optical (NLO) materials is extremely promising. In initial reports, the chemical flexibility of the sol-gel approach has enabled researchers to incorporate specific constituents which provide the nonlinear susceptibility (conjugated polymers, organic molecules, semiconductor particles), within an essentially inorganic matrix which provides superior stability, optical properties and fabrication capability.

Two different sol-gel approaches have been used to incorporate conducting polymers into sol-gel matrices. The high degree of $\pi$ conjugation found in conducting polymers makes them attractive candidates for third order NLO materials because of the extended network of delocalized $\pi$ electrons. Although conjugated polymers have demonstrated large $\chi^{(3)}$ coefficients, they generally exhibit poor processing characteristics and lack environmental stability. Prasad and co-workers have successfully synthesized silica gel/conjugated polymer composites containing up to $50 \%$ (by weight) polymer. ${ }^{43}$ ) They used a water soluble precursor of the conjugated polymer, poly-p-phenylene vinylene (PPV), mixing it with the silica gel precursor and a common solvent. Upon heating to temperatures above $140^{\circ} \mathrm{C}$, the PPV precursor was converted to form the conjugated polymeric structure. High optical quality as-cast films were synthesized and a number of optical waveguide studies have been performed. At 1.06 $\mu \mathrm{m}, \chi^{(3)}$ of $\approx 3 \times 10^{-10}$ esu was measured by the degenerate four-wave mixing (DFWM) technique. Not only is this value comparable to those of conducting polymers, but the nonlinear response time is in the subpicosecond range. Prasad also reported the fabrication of two-dimensional gratings using the silica/ PPV films and demonstrated the feasibility of using these films for optical recording. ${ }^{43)}$

Another sol-gel approach has been used to synthesize polyaniline-doped silica gels. ${ }^{44)}$ Polyaniline (or PANi) was selected because it can be made highly soluble in a variety of solvents. The PANi guest was incorporated into a silica sol host by dissolving the polymer into a mutually compatible solvent, $\mathrm{N}$ methyl pyrrolidinone (NMP). This characteristic was used to prepare silica gel monoliths containing PANi or 2-ethyl PANi. ${ }^{45)}$ The resulting material contained substantially less of the conjugated polymer $\left(\approx 10^{-3} \mathrm{M}\right.$ ) than the films obtained by polymerizing PPV. Despite the low concentration, DFWM measurements indicated that $\chi^{(3)}$ for the material at 1.06 $\mu \mathrm{m}$ was $\approx 30 \%$ of the value of a $\mathrm{CS}_{2}$ reference sample (i.e., $\chi^{(3)} \approx 5 \times 10^{-13} \mathrm{esu}$ ). Since nanosecond pulses were used in these DFWM experiments, it is 
not yet established whether all of the $\chi^{(3)}$ response is electronic or if there is a thermal contribution.

Another method for producing NLO sol-gel materials has involved the addition of organic molecules which exhibit large nonlinear effects. Two beam and four beam techniques using an $\mathrm{Ar}^{+}$laser operating at $514 \mathrm{~nm}$ as the pump source were used to determine the diffraction efficiencies for ORMOSILs doped with methyl red, ${ }^{46)}$ and composite sol-gels doped with acridine orange or methyl orange. ${ }^{47)} \mathrm{Ap}$ propriate gratings were developed in all three materials although it is likely that thermal gratings, formed by absorption of the pump signal by the various dyes, are the source of the nonlinear response.

The doping of organic molecules in a sol-gel matrix has also been able to produce materials which exhibit second harmonic generation (i.e., $\left.\chi^{(2)}\right) .{ }^{48)}$ The surprising feature with this work is that a nonlinear molecule such as MNA (2-methyl-4-nitroaniline) requires a non-centrosymmetric environment in order to exhibit second harmonic generation (SHG). The sol-gel materials investigated in this study were fairly thick films (25 to $30 \mu \mathrm{m}$ thick) comprised of an organic modified silica gel, with the organic dye dissolved in the organic constituent, trimethoxysilylpropyl substituted polyethyleneimine (MPEI). A substantial range of compositions was investigated and although crystallization could be observed for certain regimes, a significant SHG response was obtained with compositions far from the crystallization limit.

This work by Boulton et al. implies that molecular alignment may occur in sol-gel systems. A SHG effect was also observed with pNA (p-nitroaniline) doped films. ${ }^{48)}$ In this case it is not likely that crystallization is responsible since pNA exhibits centrosymmetric crystallization. Related to this is the behavior reported for silica/PPV films. ${ }^{43)}$ The as cast materials show substantial birefringence as the refractive indices are 1.72 and 1.60 for the TE and TM modes, respectively. The prospect that sol-gel films may possess locally oriented microstructures would enable a wide range of optical properties to be designed into these materials.

The use of sol-gel techniques to produce semiconductor microcrystals doped in silica glasses has successfully demonstrated significant quantum size effects and NLO responses. ${ }^{49), 50)}$ Small particle sizes for $\mathrm{ZnS}, \mathrm{CdS}$ and $\mathrm{PbS}$ doped in silica glass have been reported recently. ${ }^{50)}$ The synthesis method involves combining acetates of the metal with TEOS and, after appropriate gelation and drying, heat treating the gel glass in $\mathrm{H}_{2} \mathrm{~S}$. Crystalline particles $<60 \AA$ were produced in this fashion. The quantum size effects were evident by the rather significant blue shifts of the absorption edge of the doped silicas as compared to the bulk crystals of $\mathrm{ZnS}, \mathrm{CdS}$ and $\mathrm{PbS}$. A $\chi^{(3)}$ value of $1.5 \times 10^{-10}$ esu was measured at $390 \mathrm{~nm}$ by DFWM for a sample of $2 \% \mathrm{CdS}$ doped silica. Other methods for producing semiconductor particles in sol-gel matrices are being actively pursued. ${ }^{51), 52)}$

Another optical property related to photonic technology is photochemical hole burning (PHB). PHB uses a site-selective photoinduced reaction to "burn a hole" in the inhomogeneously broadened absorption band of the dopant. The phenomenon has been proposed for high density optical data storage. The work to date has established that PHB can be achieved in organic-doped silica gels. Experiments with 1, 4-dihydroxyano-thraquinone ${ }^{53)}$ and tetraphenylporphinetetrasulfonic acid ${ }^{54}$ indicate that the hole burning is photochemical in nature and that the dopants are well dispersed and isolated in the silica matrix.

\subsection{Optical chemical sensors}

The overall requirements for a selective ion or molecular sensor are an indicator molecule that reversibly interacts with a target molecule with a high degree of specificity and that has some detectable property that changes when the target molecule has interacted. Among the most familiar chemical sensors are acid-base $\mathrm{pH}$ indicators that change color when the target species (hydronium or hydrox$\mathrm{yl}$ ions) interacts with the indicator. Optically based sensors are especially convenient and accurate because changes in the absorbance of the medium containing the sensor can be readily measured or even visually detected. An optical property that is suitable for remote sensing is luminescence. Changes in the wavelength, lifetime and/or intensity of the emitted light can be monitored and measured by imaging or by optical fiber transmission.

Sol-gel glasses are almost ideal host matrices for optical chemical sensors. They have the obvious advantages of chemical inertness, mechanical stability and optical transparency. More importantly, they are able to physically encapsulate the indicator molecule in pores in the glass such that these molecules are immobilized and cannot be leached out, while at the same time they are porous enough to allow transport of solvent and other small molecules or ions into the interior of the glass. The glasses can be fabricated into desired shapes and sizes such as monolithic blocks or thin films for specific applications.

Several recent observations revealed the feasibility of making sol-gel glass based optical sensors. During our studies of pyranine as a probe of chemical changes occurring during gelation and drying, we discovered that the pyranine probe molecule could respond to the surrounding environment. ${ }^{23)}$ Most importantly, once the samples were dried, we found that they can easily reabsorb water vapor from the air or from liquids. Gas and liquid diffusion into the xerogel was very rapid due to the interconnecting pores which represent approximately half of the gel volume. The emission spectra of the doped-glass responded to the ambient medium to which the gel 
was exposed. Avnir et al. found that the luminescence of molecular dopants in sol-gel glasses could be quenched by molecules and gases surrounding the glass. ${ }^{55}$ ) The exciting outgrowth of these observations is the feasibility of making glasses having optical properties which change in the presence of target molecules, i.e. of making sol-gel glass chemical sensors.

\section{$\mathrm{pH}$ sensors}

The simplest chemical sensors which have been demonstrated to date are $\mathrm{pH}$ sensors because the response to the target ion is an easily recognizable color change. The discovery that initiated these studies was the response of pyranine to external solutions as described above. Pyranine-doped glasses reversibly changed color when exposed to acidic and basic aqueous solutions. These observations showed that the large indicator molecule is trapped in the matrix whereas small molecules can diffuse into the matrix. Thymophthaleine, ${ }^{56)}$ phenolphthaleine ${ }^{56)}$ and phenol red ${ }^{57)}$ have been doped into TEOS based gel-glasses. When the glass is immersed in appropriate acid-base solutions, the glasses change color at the same $\mathrm{pH}$ values as do the indicators in solutions of the same $\mathrm{pH}$. Response times are typically fast, on the order of $1 \mathrm{sec}$ for the phthaleines. Films having thicknesses on the order of several thousand angstrom have been spin coated on glass substrates. ${ }^{57)}$ The films doped with acid-base indicators reversibly respond to $\mathrm{pH}$ in less than one second.

\section{Metal ion sensors}

Gel-glasses have been made which respond to the presence of metal ions. All of the sensors reported to date have been studied in a very preliminary fashion, and details about the reversibility and response times are generally lacking. In some cases, colorimetric reagents such as dimethylglyoxime (for $\mathrm{Ni}^{2+}$ ) and o-phenanthroline (for $\mathrm{Fe}^{2+}$ ) have been incorporated in silica glasses and have been shown to produce the characteristic color when exposed to the metal (red for both $\mathrm{Ni}^{2+}$ and $\mathrm{Fe}^{2+}$ ). ${ }^{56}$ ) In other cases, a metal ion (e.g., iron) has been trapped and the glass has been exposed to solutions containing hexacyanoferrate to produce intensely colored Prussian blue. ${ }^{57)}$ When monoliths having dimensions of $2 \times 2 \times 6 \mathrm{~mm}$ were exposed and then later removed from the solution, washed and cleaved, prussian blue was found to have formed in the interior of the monolith.

\subsection{Photochromic gels and glasses}

Photochromism is defined as the reversible lightinduced color change caused by absorption of a photon. Photochromic glasses have several important uses depending on the rates of the transformations. For example, if the transformations are fast, the photochromic glasses can be used as optical switches. Light of one color causes the change in the absorption spectrum of the glass which, in turn, transmits or blocks light of a second color. If the transformations are very slow (on the order of years), then the photochromic glasses can be used as optical data storage media. Light of one color is used to "write" on the glass and light of a second color is used to "read" the stored information. In addition to the technological applications, photochromic glass can be used to form energy conserving coatings, eye protection glasses, and privacy shields. Current research is focussed on making new photochromic glasses by encapsulating photochromic molecules in gel-glasses. The color changes and the rates depend on both the nature of the encapsulated molecules and the interaction of the molecules with the glass.

\section{Aluminosilicate gels}

Three derivatives of the photochromic 1', 3', 3'trimethylspiro[2H-1-benzopyran-2, 2'-indoline molecule, abbreviated BIPS, were studied in our laboratories. ${ }^{58)}$ They are PNM-BIPS, 1'-phenyl, 6nitro, 8-methoxyBIPS; PN-BIPS, 1'-phenyl, 6-nitro BIPS; and CNM-BIPS, 1'-chloro, 6-nitro, 8-methoxy BIPS. All three showed reversible photochromism in aluminosilicate gels. Of these, PNM-BIPS was the most stable in the gels and gel-glasses and it was studied in the most detail. Freshly prepared gels containing PNM-BIPS were clear and highly photochromic. Aging of the gels did not significantly change the photochromic properties. Photochromic transparent solids of dimensions of $1 \times 1 \times 1 \mathrm{~cm}$ were easily prepared and studied.

Transient absorption spectra for an aged gel containing PNM-BIPS are shown in Fig. 9. Both the growth of the absorption in the visible during irradiation with the UV pump beam and the decay of the ab-

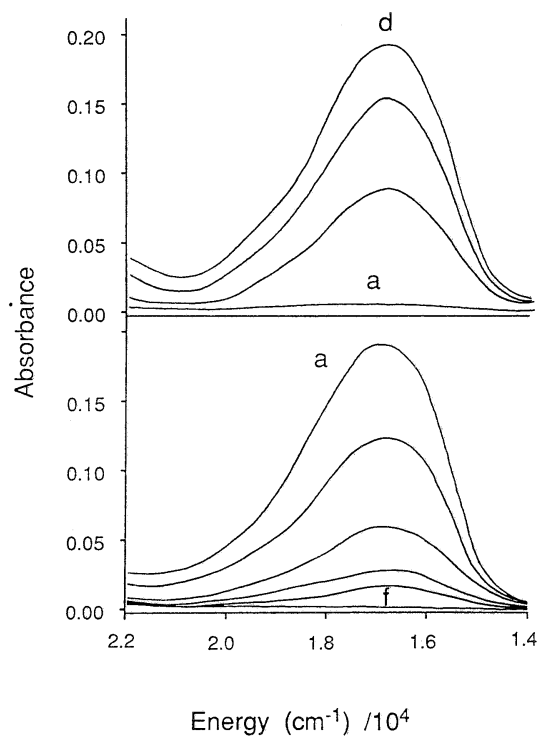

Fig. 9. Transient absorption spectra for a sealed ASE gel containing PMN-BIPS. Upper curve: Growth of the transient absorption band at $16950 \mathrm{~cm}^{-1}$ during irradiation with a UV excitation source. Lower curve: Decrease in the absorbance of the transient upon cessation of the excitation. Transient absorption spectra are shown at times (a) 0,2,6, (d) 9, 12 and (f) 45 seconds after the pump beam was blocked. 
sorption after the pump beam is blocked are shown. In the spectral range examined, $22000 \mathrm{~cm}^{-1}$ to $14000 \mathrm{~cm}^{-1}$, the spectrum is dominated by a single band growing in at $16950 \mathrm{~cm}^{-1}$. At the high energy end of the $16950 \mathrm{~cm}^{-1}$ band the tail of the second band can be seen. When the pump beam is unblocked (Fig. 9a), the band at $16950 \mathrm{~cm}^{-1}$ grows in rapidly, reaching a maximum absorbance of about 0.2 in about twelve seconds. Upon blocking the pump beam (Fig. 9b) the band at $16950 \mathrm{~cm}^{-1}$ disappears over several seconds to leave the net zero transient absorption seen before the pump beam was unblocked. The total disappearance of the transient absorption band shows that the photochromism is reversible in the gel. The reversibility exists in gels 3 months old. The violet color of the transient species results from the higher transmittance in the blue region and a smaller transmittance in the red region.

The rate at which the transient absorbing species is formed and subsequently reverts back to the starting material was monitored near the maximum of the transient absorption spectrum at $15803 \mathrm{~cm}^{-1}$ by using a He-Ne laser. In all cases the absorbance at $15803 \mathrm{~cm}^{-1}$ was low for the unexcited gels. Upon excitation with a mercury lamp at wavelengths shorter than $4000 \AA$, the absorbance at $15803 \mathrm{~cm}^{-1}$ rapidly rises and then levels off as the irradiation continues. When the excitation ceases, the absorption at 15803 $\mathrm{cm}^{-1}$ gradually decreases. A plot of the transmittance at $15803 \mathrm{~cm}^{-1}$ of a PNM-BIPS gel is shown in Fig. 10. The plot shows the baseline transmittance before the pump (A), the increase in the absorbance when the pump is unblocked (A-B), and the decrease in the absorbance after the pump is blocked $(\mathrm{B}-\mathrm{C})$. The figure shows the results for a 7 day old gel.

A plot of the natural log of the absorbance as a function of time after the excitation ceased (point $\mathrm{C}$ ) is linear. The exact form of the $\ln \mathrm{A}$ versus time plot is sensitive to the choice of the baseline. The ex-

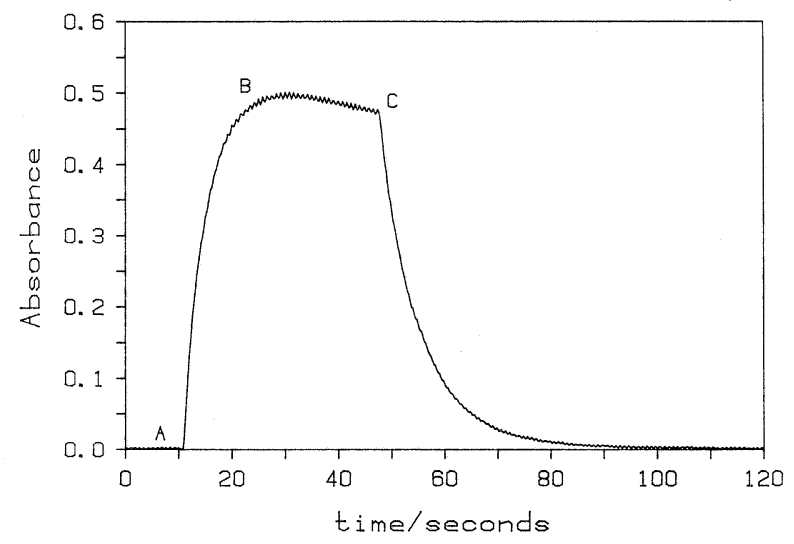

Fig. 10. The absorbance at $15803 \mathrm{~cm}^{-1}$ for an ASE gel containing PNM-BIPS. The excitation of the sample began at point $A$ and continued through part $\mathrm{B}$ to part $\mathrm{C}$ at which time the excitation ceased. The decay of the absorption of the transient follows point $\mathrm{C}$. perimental uncertainty in determining the slopes is about $\pm 10 \%$.

During the initial gelation of the aluminosilicate, no change in the rates of the rise or decay of the $16950 \mathrm{~cm}^{-1}$ absorption caused by the photointermediate is observed. As the gels age over a period of about 100 days, the lifetimes of the photointermediates slightly increase but do not undergo major changes. The lifetime is approximately 5 seconds. The solid gels are fully photochromic, i.e. the ringopening reaction is not a sensitive probe of the polymerization and aging processes. In contrast, the rigidochromic probe of the microscopic rigidity of the medium clearly indicated that the oxide skeleton becomes increasingly rigid during aging. ${ }^{20)}$

Gels which remain sealed over several months retain their photochromic properties, although there is an apparent reduction in the intensity of the color after many weeks. For all three BIPS tested, the lifetime of the species absorbing at $15803 \mathrm{~cm}^{-1}$ did not change in sealed gels over several months. Since the lifetime of the merocyanine form of the spiropyran is longer at higher viscosities, this result implies that the viscosity in sealed gels did not change significantly over several months. As long as evaporation of the solvent and polymerization products is prevented, reversible photochromism is exhibited in the gels.

Once evaporation starts, no change in rate constants is evident until some $70 \%$ weight loss has occurred. As drying progresses, the gel structure collapses and the pore volume decreases. The pores, however, are relatively large (50 to $100 \AA$ ) and, therefore, it is not likely that the kinetics are being affected by physical restrictions imposed by the gel structure. Instead, the increase in rate constant must be associated with the changes which occur with solvent loss. One possibility is that the BIPS molecule interacts with the wall of the pore. This interaction could then inhibit the ring-opening mechanism which is of fundamental importance to the photochromic process. Another possibility is that the effective viscosity of the solvent increases as the fraction of solvent phase in the pores becomes small and there is just a thin film of liquid covering the pore wall. As previous studies have indicated, a viscosity increase can lead to an increase in the rate constant. 59) The increase in the lifetime of the intermediate during drying provides a sensitive probe of the changes occurring during the drying process. It is interesting to note that drying to a weight loss of about $75 \%$ causes both the photochromism to cease and the rigidochromic emission ${ }^{20)}$ to level out at its maximum energy.

Silicon dioxide and modified silicon dioxides

Photochromic BIPS molecules have been incorporated in $\mathrm{SiO}_{2}$ and various chemically modified $\mathrm{SiO}_{2}$ matrices. ${ }^{60), 61)}$ The properties of the unmodified $\mathrm{SiO}_{2}$ xerogels were similar to those of the aluminosilicate xerogels; the photochromism stopped and these 
materials can not be used for practical applications. However, BIPS molecules incorporated in modified silica xerogels prepared from $\mathrm{Si}\left(\mathrm{OC}_{2} \mathrm{H}_{5}\right)_{3} \mathrm{CH}_{2} \mathrm{CH}_{3}$ and from the copolymerization of TMOS with polydimethylsiloxanes under mildly basic conditions retained their activity even at the dry stage. In the case of the former xerogel, normal photochromism, i.e., a change from colorless to colored upon irradiation, was observed. In the latter case, materials with reversed photochromism (colored to colorless) were obtained. The difference between the normal and the reversed photochromism was explained in terms of the differences in the properties of the pores in which the BIPS molecule is trapped. In the case of the normal photochromism, the pore has an apolar surface composed of $\mathrm{Si}-\mathrm{CH}_{2} \mathrm{CH}_{3}$ groups which do not stabilize the colored form, whereas in the case of the reversed photochromism, the pore surface consists of $\mathrm{Si}-\mathrm{OH}$ groups and is similar to that of $\mathrm{SiO}_{2}$ glasses.

\section{Summary and conclusions}

It is now well established that the sol-gel approach is a powerful means for producing organic-doped photonic materials. By using appropriate precursors and solvents, researchers have demonstrated that a wide variety of organic molecules and polymers can be incorporated in sol-gel matrices. The work to date has, in general, evolved towards two different objectives. One direction has been to use the organic dopant as a luminescent probe of the sol-gel process. In this role, the probe molecules furnish substantial insight regarding local sol-gel chemistry and structure. These probes provide detailed information about gelation and aging at a time in the sol-gel process when traditional solution and solid-state methods are not as useful. Moreover, the ability to do control experiments in solution as a reference point for interpreting the spectroscopic response of the probe molecules in gels provides a sound scientific basis for this work.

The second direction of research, that of the deliberate doping of organics in sol-gels, is clearly emerging as an important means of developing new photonic materials. It is now evident that dye molecules, photochromic molecules and even conducting polymers are able to retain their specific optical properties in sol-gel matrices. Within a relatively short period of time many significant results have been achieved in the areas of tunable solid-state lasers, third order susceptibility and photochromic behavior. A new generation of optically-based chemical sensors based on the tailoring of gel porosity is likely to be the source of the next dramatic results in this area.

Organic-doped sol-gel glasses offer a unique opportunity for photonic materials; that of providing the desired and deliberately chosen response of organic constituents in an essentially inorganic host matrix which possesses superior chemical and thermal stability, optical properties and fabrication capability. The emergence of photonics technology ensures that this remarkable approach for synthesizing solidstate optical materials will be the subject of continued research and development in the years ahead.

Acknowledgments The authors are grateful for the support of this work by the National Science Foundation (DMR 9003080). Additional support has been furnished by Lockheed Corp. under the UC MICRO program. We greatly appreciate the many contributions of our students and colleagues: J. Altman, P. Fuqua, R. Kaner, E. Knobbe, J. McKiernan, W. Nie, F. Nishida, S. Parveneh, J. C. Pouxviel, D. Preston, O. Stafsudd and S. Yamanaka.

\section{References}

1) For a general review see the following:

Proc. Mater. Res. Soc. Symp., "Better Cermics Through Chemistry," Vol. 32 (1984), Vol. 73 (1986), Vol. 121 (1988), Vol. 180 (1990), Ed. by C. J. Brinker, D. E. Clark and D. R. Ulrich; "Ultrastructure Processing of Advanced Cermics", Ed. by J. D. Mackenzie and D. R. Ulrich, John Wiley, New York (1988).

2) L. L. Hench and J. K. West, Chem. Rev., 90, 33 (1990).

3) L. C. Klein, Ed. "Sol-Gel Technology", Noyes Publications, Park Ridge, N. J. (1988).

4) L. C. Klein, Ann. Rev. Mater. Sci., 15, 227 (1985).

5) S. Sakka and K. Kamiya, J. Non-Cryst. Solids, 42, 403 (1980).

6) D. Avnir, D. Levy and R. Reisfeld, J. Phys. Chem., 88, 5956 (1984).

7) D. Avnir, V. Kaufman and R. Reisfeld, J. Non-Cryst. Solids, 74, 395 (1985).

8) A. Makishima and T. Tani, J. Am. Ceram. Soc., 69, C-72 (1986).

9) E. J. A. Pope and J. D. Mackenzie, MRS Bulletin, 12, 29 (1987).

10) Y. Kobayashi, Y. Imai and Y. Kurokawa, J. Mater. Sci. Lett., 7, 1148 (1988).

11) C. Sanchez, "Proc. SPIE Conf. on Sol-Gel Optics", Ed. by J. D. Mackenzie and D. R. Ulrich, Vol. 1328, SPIE, Bellingham, WA (1990) pp. 40-51.

12) M. Genet, V. Brandel, M-P Lahalle and E. Simoni, $C$. $R$. Acad. Sci. Paris, 311, 1321 (1990).

13) C. A. Capozzi and L. D. Pye, Proc. SPIE, 970, 135 (1988).

14) E. T. Knobbe, B. Dunn, P. D. Fuqua and F. Nishida, $A p p l$. Optics, 29, 2729 (1990).

15) M. D. Newsham, M. K. Cerrata, K. A. Berglund and D. G. Nocera, Mater. Res. Soc. Symp. Proc., 121, 627 (1988).

16) J. M. McKiernan, S. A. Yamanaka, E. T. Knobbe, J. C. Pouxviel, S. Parveneh, B. Dunn and J. I. Zink, J. Inorg. and Organomet. Polymers, 1, 87 (1991).

17) J. C. Pouxviel, S. Parvaneh, E.T. Knobbe and B. Dunn, Solid State Ionics, 32/33, 646 (1989).

18) M. Genet, V. Brandel, M. D. Lahalle and E. Simoni, "Proc. SPIE Conf. on Sol-Gel Optics", Ed. by J. D. Mackenzie and D. R. Ulrich, Vol. 1328, SPIE, Bellingham, WA (1990) pp. 194-200.

19) R. Reisfeld, M. Eyal and D. Brusilovsky, Chem. Phys. Lett., 153, 210 (1988).

20) J. McKiernan, J. C. Pouxviel, B. Dunn and J. I. Zink, J. Phys. Chem., 93, 2129 (1989).

21) a) M. S. Wrighton and D. L. Morse, J. Am. Chem. Soc., 96, 998, (1974).

b) P. J. Giordano and M. S. Wrighton, J. Am. Chem. Soc., 101, 2888 (1979).

22) J. C. Pouxviel, J. P. Boilot, A. Lecomte and A. Dauger, $J$. Phys. (Paris), 48, 921 (1987).

23) J. C. Pouxviel, B. Dunn and J. I. Zink, J. Phys. Chem., 93 , 
2134 (1989).

24) J. C. Pouxviel, Ph. D. Thesis, University of Paris VI (1987)

25) V. R. Kaufman, S. C. Muller, K. H. Muller and D. Avnir, Chem. Phys. Lett., 142, 551 (1987).

26) a) N. R. Clement and M. Gould, Biochemistry, 20, 1534 (1981).

b) K. Kano and J. H. Fendler, Biochim. Biophys. Acta, 509 289 (1978).

27) V. R. Kaufman, D. Avnir, D. Pines-Rojanski and D. Huppert, J. Non-Cryst. Solids, 99, 379 (1988).

28) V. R. Kaufman and D. Avnir, Langmuir, 2, 717 (1986).

29) K. Matsui, T. Nakazawa and H. Morisaki, J. Phys. Chem., 95, 976 (1991).

30) V. V. Rodchenkova et al., Opt. Spectrosc. (USSR), 60, 35 (1986).

31) G. B. Altshuler et al., Opt. Spectrosc. (USSR), 62, 709 (1988).

32) Y. Kobayashi, Y. Kurokawa and Y. Imai, J. Non-Cryst. Solids, 105, 198 (1988).

33) B. Dunn, E. Knobbe, J. McKiernan, J. C. Pouxviel and J. I. Zink, Mater. Res. Soc. Symp. Proc., 121, 331 (1988).

34) F. Salin, G. LeSaux, P. Georges, A. Brun, C. Bagnall and J. Zarzycki, Opt. Letts., 14, 785 (1989).

35） R. Reisfeld, D. Brusilvsky, M. Eyal, E. Miron, Z. Burstein and J. Ivri, Chem. Phys. Lett., 160, 43 (1989).

36) J. M. McKiernan, S. A. Yamanaka, B. Dunn and J. I. Zink, J. Phys. Chem., 94, 5652 (1990).

37) R. Reisfeld, D. Brusilovsky, M. Eyal, E. Miron, Z. Burstein and J. Ivri, Proc. SPIE, 1182, 230 (1989).

38) J. C. Altman and R. E. Stone, unpublished results.

39) J. C. Altman, R. E. Stone, B. Dunn and F. Nishida, Photonics Tech. Lett., 3, 189 (1991).

40) G. Jones, W. R. Jackson and A. M. Halpern, Chem. Phys. Lett., 72, 391 (1980).

41) R. Reisfeld, "Proc. SPIE Conf. on Sol-Gel Optics", Ed. by J. D. Mackenzie and D. R. Ulrich, SPIE, Bellingham, WA (1990) pp. 29-39.

42) B. Dunn, F. Nishida, J. C. Altman and R. E. Stone, "Ultrastructure Processing of Ceramics”, Ed. by L. L. Hench and J. K. West, Wiley, New York, in press.

43) P. N. Prasad, Mater. Res. Soc. Symp. Proc., 180, 741 (1990).

44) J. I. Zink, B. Dunn, R. B. Kaner, E. T. Knobbe and J. McKiernan, "Materials For Nonlinear Optics-Chemical Perspectives", Chap. 36, Ed. by S. R. Marder, J. E. Sohn and G. D. Stucky, American Chemical Society, Washington (1991) pp. 541-52.

45) F. Nishida, B. Dunn, E. T. Knobbe, P. D. Fuqua, R. B. Kaner and B. R. Mattes, Mater. Res. Soc. Symp. Proc., 180, 747 (1990).

46) E. T. Knobbe, B. Dunn, P. Fuqua, F. Nishida and J. I. Zink, "Ultrastructure Processing of Ceramics", Ed. by D. R. Uhlmann et al., Wiley, New York, in press.

47) R. Reisfeld and C. K. Jorgensen, in "Structure and Bonding", Springer-Verlag, Berlin (1991) in press.

48) J. M. Boulton, J. Thompson, H. H. Fox, I. Gorodisher, G. Teowee, P. D. Calvert and D. R. Uhlmann, Mater. Res. Soc. Symp. Proc., 180, 987 (1990).

49) M. Nogami, K. Nagasaka and E. Kato, J. Am. Ceram. Soc., 73, 2097 (1990).

50) M. Nogami, M. Watabe and K. Nagasaka, "Proc. SPIE Conf. on Sol-Gel Optics", Ed. by J. D. Mackenzie and D. R. Ulrich, Vol. 1328, SPIE, Bellingham, WA (1990) pp. 11924.

51) N. Tohge, M. Asuka and T. Minami, "Proc. SPIE Conf. on Sol-Gel Optics", Ed. by J. D. Mackenzie and D. R. Ulrich, Vol. 1328, SPIE, Bellingham, WA (1990) pp. 125-32.

52) C. M. Bagnall and J. Zarzycki, "Proc. SPIE Conf. on Sol-Gel Optics", Ed. by J. D. Mackenizie and D. R. Ulrich, Vol. 1328, SPIE, Bellingham, WA (1990) pp. 108-18.

53) T. Tani, H. Namikawa, K. Arai and A. Makishima, J. Appl. Phys., 58, 3559 (1985)

54) A. Makishima, K. Morita, H. Inoue and T. Tani, "Proc. SPIE Conf. on Sol-Gel Optics", Ed. by J. D. Mackenzie and D. R. Ulrich, Vol. 1328, SPIE, Bellingham, WA (1990) pp. 264-67.

55) A. Slama-Schwok, D. Avnir and M. Ottolenghi, J. Phys. Chem., 93, 7544 (1989).

56) R. Zusman, C. Rottman, M. Ottolenghi and D. Avnir, J. NonCryst. Solids, 122, 107 (1990).

57) J. I. Zink, B. Dunn, and S. Yamanaka, unpublished results

58) D. Preston, T. Novinson, W. C. Kaska, B. Dunn and J. I. Zink, J. Phys. Chem., 94, 4167 (1990).

59) R. C. Bertelson, in Techniques of Chemistry, Vol. III: "Photochromism", G. H. Brown, Ed., Wiley-Interscience, New York (1971) Chapter VII, p. 100.

60) D. Levy and D. Avnir, J. Phys. Chem., 92, 4737 (1988).

61) D. Levy, S. Einhorn and D. Avnir, J. Non-Cryst. Solids, in press. 


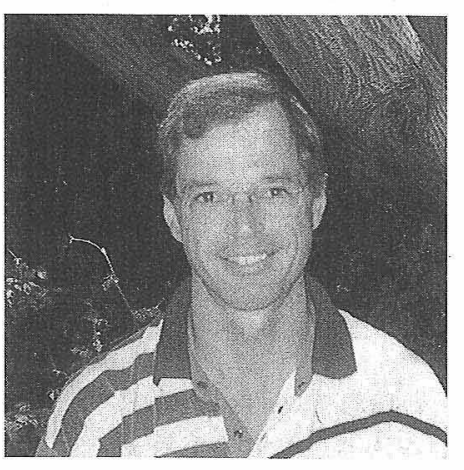

Jeffrey I. ZINK received his B.S. from University of Wisconsin, 1966, and Ph. D. degree from the University of Illinois in 1970. He joined the faculty of the Department of Chemistry and Biochemistry at UCLA in the summer of 1970. His research interests, in addition to the study of new optical materials made by encapsulating organic and organometallic molecules in sol-gel glasses, include single crystal polarized electronic spectroscopy of inorganic molecules, the determination of distortions in excited electronic states by using electronic spectroscopy, resonance Raman spectroscopy and time-dependent theory, triboluminescence (the emission of light caused by application of mechanical stress to crystals), and laser-assisted MOCVD of metal films on semiconductor substrates. The collaborative work on sol-gel materials with Professor Dunn has resulted in discoveries discussed in this paper. In addition, a new class of optically based chemical sensors is being developed. Professor Zink has published over 120 papers in his fields of interest. He has been a Camille and Henry Dreyfus Teacher Scholar (1975), an Alexander von Humboldt fellow at the University of Regensburg (1979), and a John Simon Guggenheim Fellow (1988). He has won the Herbert Newby McCoy award (1982) and the Glenn T. Seaborg Award (1983). He is a member of the American Chemical Society, the National Audubon Society, and the honorary societies Phi Kappa Phi and Phi Lambda Epsilon.

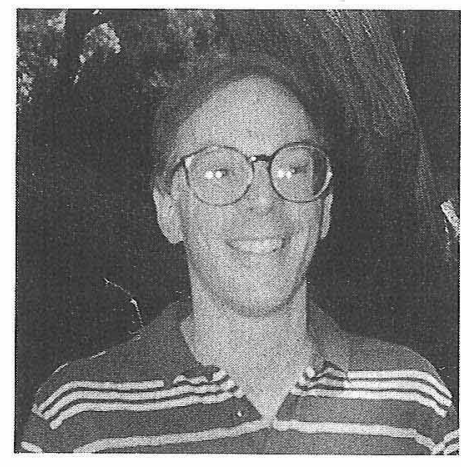

Bruce S. DUNN B.S. from Rutgers University (Ceramic Science) in 1970, M.S. from University of California, Los Angeles (Materials Science) in 1972, and Ph. D. from University of California, Los Angeles (Ceramics) in 1974. He joined the staff of General Electric Corporate Research and Development in 1976. His activities were primarily related to the sodium-sulfur battery and other electrochemical devices. His research efforts at General Electric included solid electrolytes, ceramic-to-metal seals and the kinetics of electrochemical interfaces. His work on solid electrolyte interfaces was able to establish the nature of the high temperature interfacial reactions which lead to severe polarization effects in electrochemical devices. Dr. Dunn joined the faculty of the Department of Materials Science and Engineering at UCLA in November, 1980. His current research interests involve the synthesis of ceramics and inorganic compounds and characterization of their electrical and optical properties. The materials presently being studied in his group include solid electrolytes, sol-gel materials, infrared transmitting solids and high temperature superconductors. He and his colleagues have recently been investigating the optical properties of the solid electrolyte, $\beta^{\prime \prime}$ alumina, which can exhibit high optical gain, lasing, phase conjugation and wave guiding. The work on sol-gel materials involves an investigation of the optical properties produced by incorporating a variety of different organic and organometallic molecules in the gel. The materials show considerable promise for use as sensors and optical components. Dr. Dunn has authored or co-authored over 70 publications in his fields of work and holds 12 patents. During 1985-86 he received a Fulbright research fellowship and was an Invited Professor at the University of Paris. He was promoted to Professor in 1985 and served as the Chairman of the Department of Materials Science and Engineering from 1987 to 1990 . He is a member of the American Ceramic Society, the Materials Research Society and the Electrochemical Society and presently serves on the editorial boards of the Journal of Materials Chemistry, Chemistry of Materials and Advanced Ceramic Reports. 